\title{
Undifferentiated pleomorphic sarcoma of skin in unusual locations: Report of two cases
}

\author{
Ali Baş, $\mathrm{MD}^{1}$ (D), Lercan Aslan, $\mathrm{MD}^{1}{ }^{1}$, İbrahim Levent Eralp, $\mathrm{MD}^{2}$ (D) \\ 1Department of Orthopedics and Traumatology, Koç University Hospital, Istanbul, Turkey \\ 2Department of Orthopedics and Traumatology, Istanbul University, Retired Instructor, Turkey
}

Sarcomas are rare heterogeneous group of malignancies, arising from mesenchymal-derived cells and accounting for less than $1 \%$ of all malignancies. ${ }^{[1]}$ To date, for soft tissue sarcoma (STS), more than 80 malignant histological types and subtypes, capable of metastasis and local recurrence, have been identified. About $10 \%$ of STS occur in the lower extremity. ${ }^{[2]}$

Undifferentiated pleomorphic sarcoma (UPS) was previously considered a subtype of malignant fibrous histiocytoma (MFH), until the update of the World Health Organization classification in 2013. Currently, UPS is identified as a pleomorphic malignant tumor without specified histological features. ${ }^{[3,4]}$ Superficially located UPS of the skin is a rarer entity than other malignant skin tumors. The true incidence is unclear, due to the consideration of these lesions under the name of MFH or superficial MFH. ${ }^{[5,6]}$

Received: September 10, 2020

Accepted: November 02, 2020

Published online: January 06, 2021

Correspondence: Lercan Aslan, MD. Koc Üniversitesi Hastanesi Ortopedi ve Travmatoloji Bölümü, 34010 Topkapı, Istanbul, Türkiye.

E-mail: lercan.aslan86@gmail.com

Doi: 10.5606/ehc.2021.78856

Citation: Bas A, Aslan L, Eralp IL. Undifferentiated pleomorphic sarcoma of skin in unusual locations: Report of two cases. Jt Dis Relat Surg 2021;32(1):253-257.

(2021 All right reserved by the Turkish Joint Diseases Foundation

This is an open access article under the terms of the Creative Commons Attribution-NonCommercial License, which permits use, distribution and reproduction in any medium, provided the original work is properly cited and is not used for commercial purposes (http://creativecommons.org/licenses/by-nc/4.0/).

\section{ABSTRACT}

Undifferentiated pleomorphic sarcoma (UPS) of the skin is a rare soft tissue sarcoma subtype with a high risk of metastasis and local recurrence. Ultraviolet exposure plays a prominent role in its etiology. Herein, we present two rare cases of UPS of the skin with an occurrence in non-ultraviolet exposed locations and discuss the need of wide resection and the identification of the depth of the tumor. Due to the resection of the skin, resulting defect was undertaken with skin grafting. One case was extending to the fascia and, after the resection, vacuum-assisted closure therapy was used for the formation of granulation tissue. No recurrence was seen after three and four years for either cases. In conclusion, UPS is a rare, aggressive malignant tumor with a high local recurrence and metastasis rate. Suspicion of malignancy in a plaque-like or nodular lesion in the skin is crucial, and local recurrence and metastasis risk is significantly correlated to the success of wide excision.

Keywords: Dermatologic oncology, ultraviolet exposure, undifferentiated pleomorphic sarcoma.

In this article, we present two rare cases of UPS of the skin with unusual locations, non-ultraviolet (UV)-damaged skin.

\section{CASE REPORT}

Case 1- A 73-year-old male patient was admitted to our outpatient clinic with a complaint of firm, painless, rapidly growing mass located at the anterior aspect of distal cruris persisting nearly for a year. The patient was first evaluated in another clinic, and excisional biopsy was performed with a preliminary diagnosis of lipoma. After the pathological result was reported as a sarcoma, the patient was referred to our clinic. Inspection revealed evident desquamation and several millimetric exulcerations over the plaque-like lesion (Figure 1). There was no history of trauma, known dermatological disease or history of systemic malignancy. Magnetic resonance imagining (MRI) 


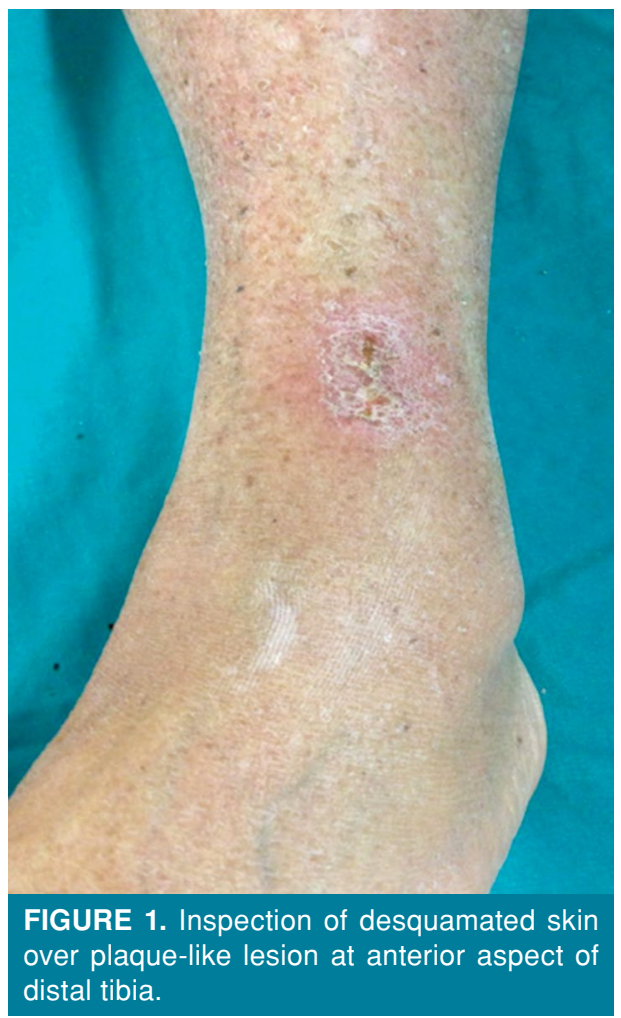

demonstrated a contrast-enhanced, $90 \times 42 \times 15-\mathrm{mm}$ skin lesion with peritumoral reactive edema, located in skin and subcutaneous fat tissue adjacent to the tibialis anterior, extensor hallucis longus, and digitorum tendons. The lesion was hypointense in $\mathrm{T} 1$ sequences and hyperintense in T2 sequences (Figure 2). Thoracic computed tomography (CT) revealed no metastasis. Surgical intervention was planned and the area of peritumoral edema was widely excised with a margin of $1-\mathrm{cm}$ tumor-free area. ${ }^{[7]}$ Intraoperative frozen-section examination revealed clean surgical margins. Following the wide resection, the exposed extensor tendons and tibialis anterior tendon and wound area were dressed by vacuum assisted closure (VAC) therapy with three-day intervals. After the achievement of adequate granulation tissue, split-thickness skin grafting (STSG) was performed (Figure 3). Gross examination revealed a $40 \times 25 \times 15-\mathrm{mm}$ sized lesion in the specimen which was, then, diagnosed as a high-grade UPS. On microscopic examination, the tumor base was closer than $1 \mathrm{~mm}$ to the deep surgical margin. Neither thoracic CT nor the ultrasound (US) scanning of regional lymph nodes (LNS) revealed any metastasis. As the surgical margin could not be achieved for the tumor base, the patient was consulted to the Multidisciplinary Tumor Board. Adjuvant radiation therapy (RT) was performed after wound healing. The patient was followed every three months for the first two years and every six months after two years using thoracic CT and cruris MRI. A three-year tumor-free survival has been achieved and the patient is still under routine follow-up. A written informed consent was obtained from the patient.

Case 2- A 73-year-old male patient was admitted to our outpatient clinic with a complaint of incidental firm mass located at the posterior aspect of the calf. A nodular skin lesion with an approximate radius of $3 \mathrm{~cm}$ was palpated over an $8-\mathrm{cm}$ induration
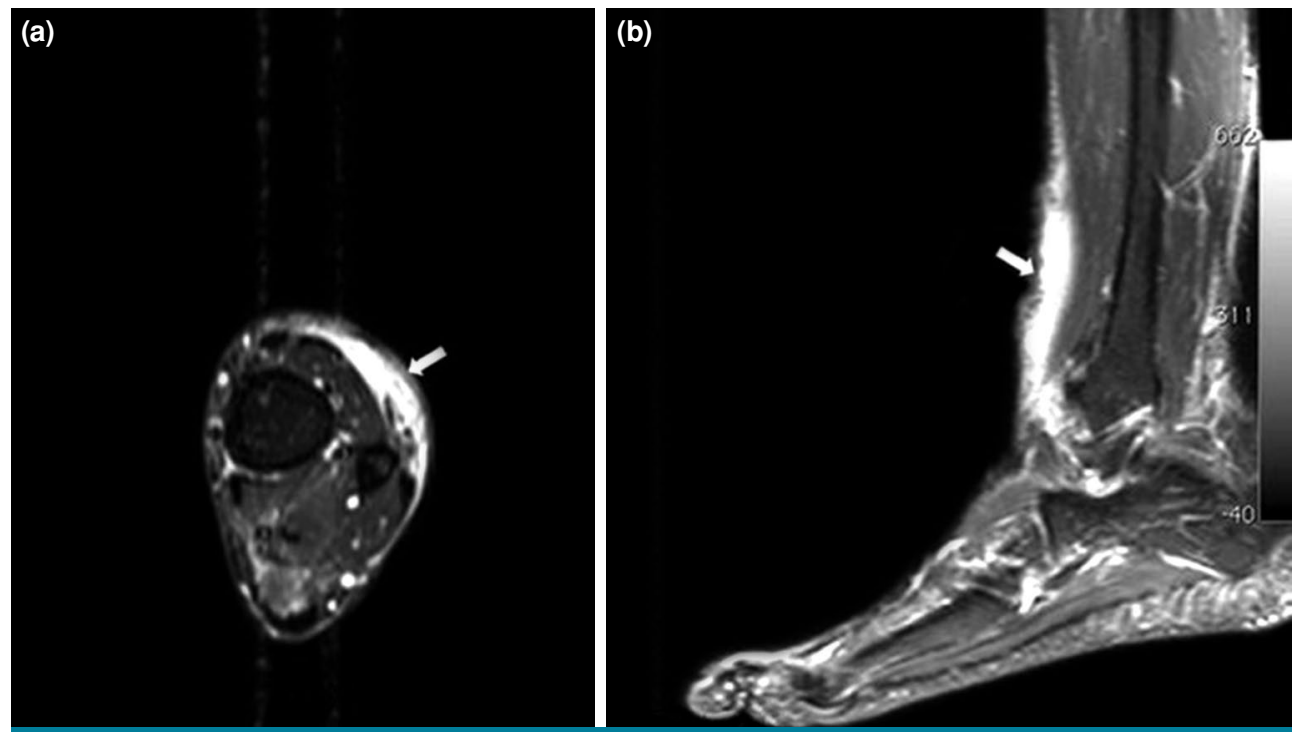

FIGURE 2. Lesion appearance on magnetic resonance imaging marked by white arrow on axial (a) and sagittal (b) planes. 

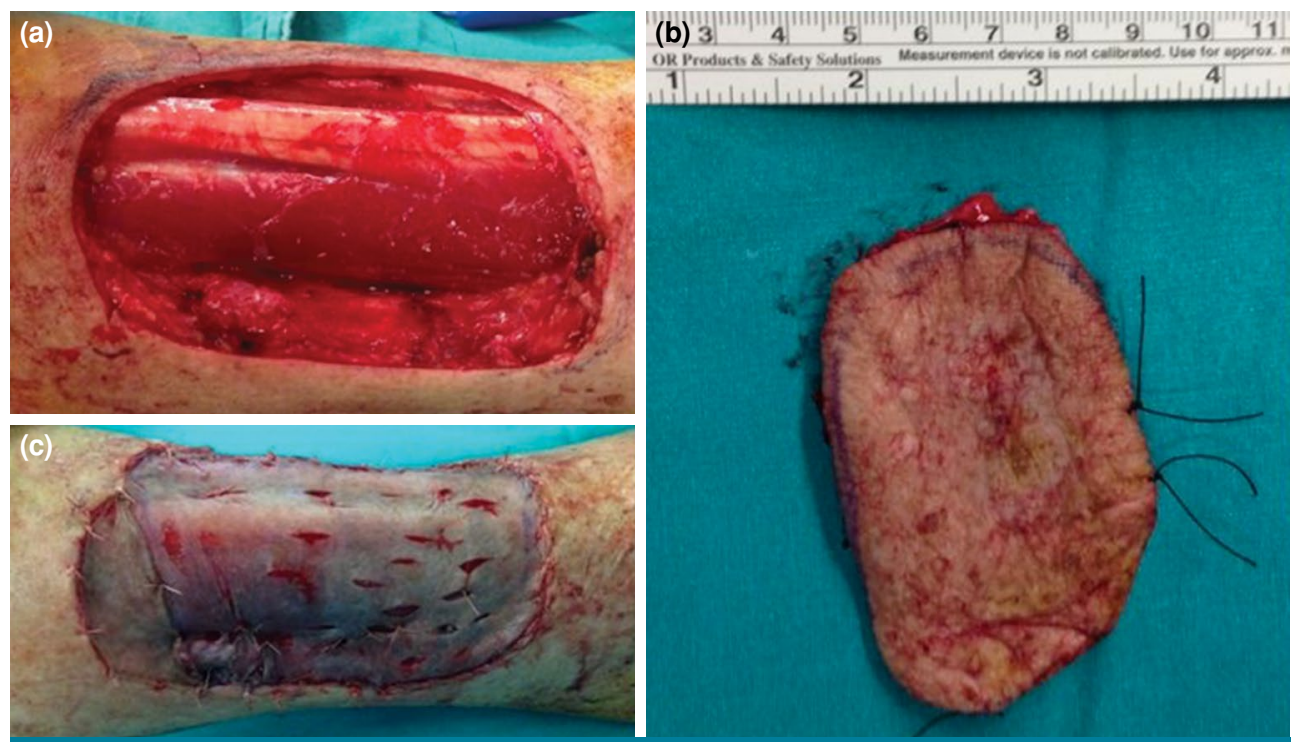

FIGURE 3. (a) Appearance after surgical resection of undifferentiated pleomorphic sarcoma. (b)Macroscopic appearance of resected specimen. (c) Split-thickness skin grafting after vacuum-assisted closure therapy.

zone. The MRI revealed an intradermally located lesion. Radiologically, the lesion was extending to the deep fascia without a clear margin (Figure 4). The Tru-cut needle biopsy resulted in pleomorphic and hyperchromatic bizarre cells infiltrating into lipocytes between collagen bands. The tumoral tissue consists of myxoid-based pleomorphic spindle cells, necrotic areas, and dense leukocyte infiltration. The mitotic index was $4 / 10$. Immunohistochemical evaluation was negative for CD34, SMA, S-100, CD31, and $\mathrm{CK}$; however, it was positive for CD68. In the light of these findings, the lesion was diagnosed as a dermal UPS or proliferative fibroblastic lesion. No metastasis was detected on thoracic CT and no popliteal or inguinal LN was detected on US. Due to the extensive peritumoral reactive edema zone and high risk of tumoral cell presence, ${ }^{[7]}$ deep fascia and adjacent gastrocnemius fibers were included in the deep surgical margin (Figure 5). Clean surgical margins were reported in the intraoperative frozensection examination. Histopathological evaluation of the $35 \times 15 \times 14-\mathrm{mm}$ sized specimen was bordered superficially by epidermis, showing a subcutaneous fat tissue invasion and necrotic areas. The pathological diagnosis was reported as UPS. As for surgical margins, the closest was $6 \mathrm{~mm}$, which was located at the base of the tumor. Surgical wound was reconstructed with STSG. The patient was followed every three months for the first two years and every six months after two years using thoracic CT and cruris MRI based on
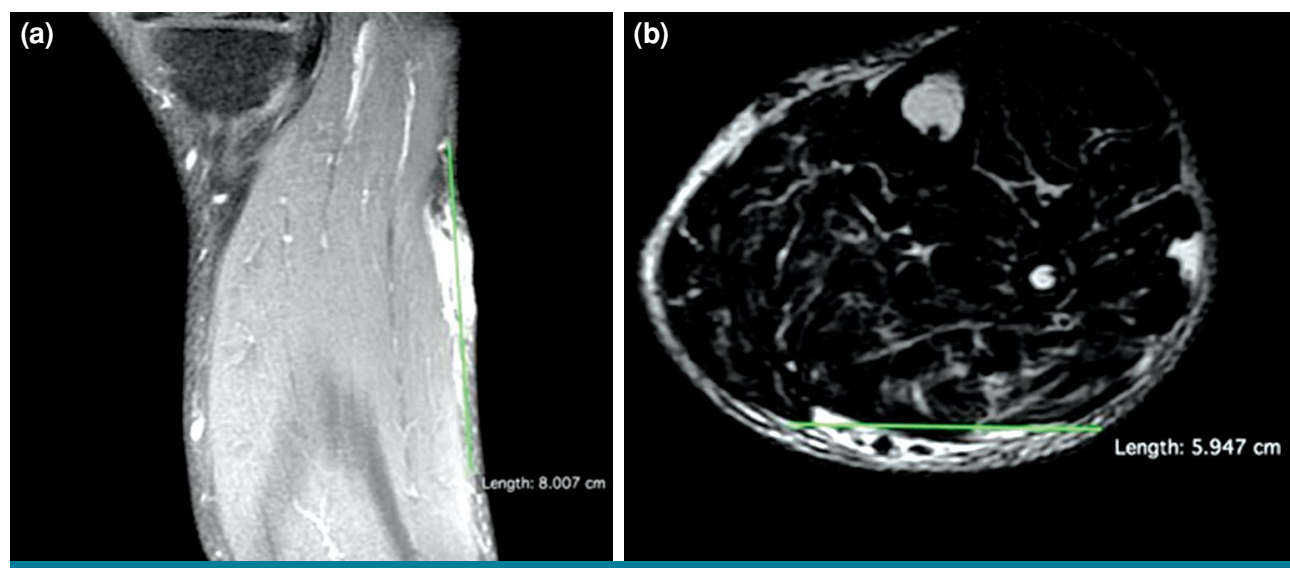

FIGURE 4. Magnetic resonance imaging showing lesion on sagittal (a) and axial (b) planes. 

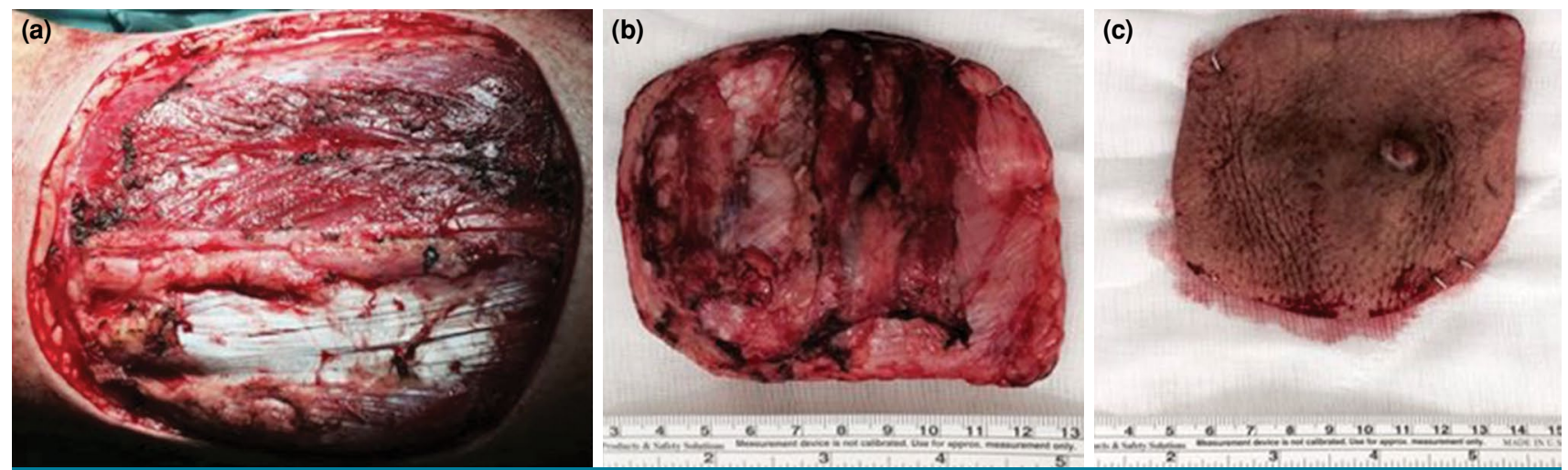

FIGURE 5. (a) Appearance after surgical resection of undifferentiated pleomorphic sarcoma. (b) Macroscopic appearance of resection base of specimen. (c) Superficial appearance of specimen with a nodular lesion located over undifferentiated pleomorphic sarcoma.

the advice of the Multidisciplinary Tumor Board. A four-year tumor-free survival has been achieved and the patient is still under routine follow-up. A written informed consent was obtained from the patient.

\section{DISCUSSION}

Undifferentiated pleomorphic sarcoma is a rare entity, which is a member of spindle cell neoplasms arising from the skin or soft tissue and can show variable clinical courses, despite being histopathologically similar. ${ }^{[8]}$ Dermal UPS is called by different names in the literature, such as atypical fibroxanthoma (AFX), superficial malignant fibrous histiocytoma (MFH), superficial UPS or pleomorphic dermal sarcoma (PDS). ${ }^{33,5,6,8]}$ Recently, to end this confusion, the term PDS was firstly recommended by Fletcher. ${ }^{[9]}$ Due to the malignant features such as subcutaneous invasion, tumor necrosis, atypical mitosis, lymphovascular and/or perineural invasion, PDS is considered to be the most aggressive in clinical and histopathological spectrum of AFX. ${ }^{[6,8,10]}$ The mutation of telomerase reverse transcriptase (TERT) is present in $76 \%$ of the skin UPS cases and appears to be associated with the UV damage. ${ }^{[6,10]}$ Thus, the increased incidence in the sun-exposed or UV-damaged skin areas such as head and neck can be explained by TERT mutation. ${ }^{[8,10]}$ On the other hand, the presence of a rapidly growing aggressive lesion on the non-UV-exposed skin areas, particularly in elderly patients, it is recommended to rule out other possible malignancies before to diagnose UPS due to its rarity. ${ }^{[10]}$ Both of the reported cases herein were located on a limited sun-exposed region of the body (i.e., the proximal calf and the anterior aspect of the distal cruris), unlike reported in the literature (i.e., the head and neck region).. ${ }^{[1]}$ Therefore, we believe that these cases are worth to be reported for their rarity and unusual locations.

In general, UPS of the skin is mostly seen around the eighth decade of life and shows a male predominance. ${ }^{[9]}$ Clinically, it manifests as usually larger than $2 \mathrm{~cm}$ in size, rapidly growing nodular or exophytic maculopapular lesion on chronically UV-damaged skin. The lesions can exhibit ulcerated and bleeding areas and, rarely, reveal a plaque-like growth pattern. ${ }^{[5,6,9]}$

Wide excision is the most common treatment method, as it is able to reveal the relationship of the tumor with skin and subcutaneous soft tissue layers and Tru-cut needle biopsy or excisional biopsy for small size tumors can be performed. ${ }^{[3,9]}$ On microscopic examination, tumor includes spindle-shaped, pleomorphic cells and atypical mitosis. ${ }^{[5,6]}$ The absence of CK, S100, CD34, and desmin expression in the immunohistochemical study are the main features of UPS of the skin for excluding the other types of sarcomas, melanomas, and carcinomas. ${ }^{[10]}$ Similarly, we performed these tests in both cases and the results were found to be negative.

Surgical resection with clear margins is the gold standard for the treatment of UPS of the skin. Although the amount of safe surgical margin in soft tissue sarcomas has not been clearly established in the literature, the positive margins are significantly associated with a high local recurrence rate. ${ }^{[3,6,9]}$ Subcutaneous adipose tissue invasion, preoperative size larger than $2 \mathrm{~cm}$, lymphovascular invasion, and immunosuppression are poor prognostic factors for local recurrence and metastasis. ${ }^{[9]}$ The risk of local recurrence and lung metastasis are encountered in up to $28 \%$ and $10 \%$, respectively. ${ }^{[6]}$ In addition, 
MRI for local staging and thoracic CT for metastasis evaluation are of utmost importance to assess the prognosis preoperatively and follow-up. Although sentinel lymph node biopsy can be preferred for squamous cell carcinomas and malignant melanomas of the skin, there is a limited number of data for UPS and prospective studies are still needed for routine use. ${ }^{[6]}$ In our cases, US was used to assess the regional lymph node metastasis before surgical excision of the tumors and no lymph node resection was required due to non-pathological features on US. A positive surgical margin is considered to be the most important prognostic factor due to the inverse relation with disease-free survival. ${ }^{[3]}$ Additionally, some other factors can cause difficulty to achieve a reliable, long-term follow-up for these patients such as being elderly, presence of systemic comorbidities related to mortality, and presence of immunosuppression as an etiological factor due to the short life expectancy. ${ }^{[9]}$

Furthermore, patients with advanced disease and those in whom negative surgical margins cannot be achieved should be discussed for adjuvant RT to decrease the local recurrence incidence; however, in the literature, RT and chemotherapy have been shown not to affect overall survival and disease-free survival. ${ }^{[3]}$

In conclusion, malignancy potential of a rapidly growing skin lesion should be taken into the consideration, and the treatment should be planned after meticulous radiological and histological examination. A multidisciplinary approach is also needed for the treatment of UPS of the skin, as for all soft tissue sarcomas. Local tumor evaluation as assessed by MRI, scanning for regional LN and distal metastasis, such as lungs, by thoracic CT should be performed preoperatively. A comprehensive histopathological evaluation and presence of positive surgical margins must be assessed for the requirement of further RT or chemotherapy.

\section{Declaration of conflicting interests}

The authors declared no conflicts of interest with respect to the authorship and/or publication of this article.

\section{Funding}

The authors received no financial support for the research and/or authorship of this article.

\section{REFERENCES}

1. Hung GY, Yen CC, Horng JL, Liu CY, Chen WM, Chen TH, et al. Incidences of primary soft tissue sarcoma diagnosed on extremities and trunk wall: A population-based study in Taiwan. Medicine (Baltimore) 2015;94:e1696.

2. Brennan MF, Antonescu CR, Moraco N, Singer S. Lessons learned from the study of 10,000 patients with soft tissue sarcoma. Ann Surg 2014;260:416-21.

3. Kamat NV, Million L, Yao DH, Donaldson SS, Mohler DG, van de Rijn M, et al. The outcome of patients with localized undifferentiated pleomorphic sarcoma of the lower extremity treated at stanford university. Am J Clin Oncol 2019;42:166-71.

4. Doyle LA. Sarcoma classification: an update based on the 2013 World Health Organization Classification of Tumors of Soft Tissue and Bone. Cancer 2014;120:1763-74.

5. Kohlmeyer J, Steimle-Grauer SA, Hein R. Cutaneous sarcomas. J Dtsch Dermatol Ges 2017;15:630-48.

6. Soleymani T, Tyler Hollmig S. Conception and management of a poorly understood spectrum of dermatologic neoplasms: Atypical fibroxanthoma, pleomorphic dermal sarcoma, and undifferentiated pleomorphic sarcoma. Curr Treat Options Oncol 2017;18:50.

7. White LM, Wunder JS, Bell RS, O'Sullivan B, Catton C, Ferguson $\mathrm{P}$, et al. Histologic assessment of peritumoral edema in soft tissue sarcoma. Int J Radiat Oncol Biol Phys 2005;61:1439-45.

8. Winchester D, Lehman J, Tello T, Chimato N, Hocker T, Kim S, et al. Undifferentiated pleomorphic sarcoma: Factors predictive of adverse outcomes. J Am Acad Dermatol 2018;79:853-9.

9. Ziemer M. Atypical fibroxanthoma. J Dtsch Dermatol Ges 2012;10:537-50.

10. Tardío JC, Pinedo F, Aramburu JA, Suárez-Massa D, Pampín A, Requena L, et al. Pleomorphic dermal sarcoma: a more aggressive neoplasm than previously estimated. J Cutan Pathol 2016;43:101-12.

11. Atik OŞ. Is there something new and interesting in my article? Eklem Hastalik Cerrahisi 2019;30:69. 